\title{
CARACTERIZACIÓN DE PACIENTES CON DISLIPIDEMIA EN UN HOSPITAL REGIONAL DE COLOMBIA
}

CHARACTERIZATION OF PATIENTS WITH DYSLIPIDEMIA AT A REGIONAL HOSPITAL OF COLOMBIA.

González Soriano Luz Stella, Lozano Espinosa Diego Alejandro², Bornachera Pinto Diego Armando³.

1 Clínica Colsubsidio Ciudad Roma, 2 Fundación Hospital Pediátrico La Misericordia, 3 Hospital Universitario San José Infantil.

Citation: González Soriano L.S., Lozano Espinosa D.A., Bornachera Pinto D. A. (2020). Caracterización de pacientes con dislipidemia en un Hospital Regional de Colombia. Revista Salud Pública y Nutrición, 19 (4), 27-36.

Editor: Esteban G. Ramos Peña, Dr. CS., Universidad Autónoma de Nuevo León, Facultad de Salud Pública y Nutrición, Monterrey Nuevo León, México. Copyright: (02020 González Soriano L.S. et al. This is an open-access article distributed under the terms of Creative Commons Attribution License [CC BY 4.0], which permits unrestricted use, distribution, and reproduction in any medium, provided the original author and source are credited.

Competing interests: The authors have declared that no competing interests exist.

DOI: https://doi.org/10.29105/respyn19.4-4

Recibido: 09 de junio 2020; $\quad$ Aceptado: 19 de octubre 2020

Email: stellags012@gmail.com 


\title{
CARACTERIZACIÓN DE PACIENTES CON DISLIPIDEMIA EN UN HOSPITAL REGIONAL DE COLOMBIA
}

\author{
González Soriano Luz Stella ${ }^{1}$, Lozano Espinosa Diego Alejandro², Bornachera Pinto Diego Armando ${ }^{3}$. \\ 1 Clínica Colsubsidio Ciudad Roma, 2 Fundación Hospital Pediátrico La Misericordia, 3 Hospital Universitario San José \\ Infantil.
}

\begin{abstract}
RESUMEN
Introducción. La dislipidemia constituye un factor de riesgo mayor y modificable en la génesis de las enfermedades cardiovasculares. Objetivo: Caracterizar los pacientes adultos con dislipidemia que consultan al servicio ambulatorio del Hospital Regional Valle de Tenza, Guateque Boyacá, Colombia, en el año 2014. Material y Método: Se realizó un estudio observacional de corte trasversal retrospectivo. Se incluyeron los pacientes de 18 o más años con alteraciones en el perfil lipídico en seguimiento por consulta ambulatoria del Hospital Regional Valle de Tenza, Guateque Boyacá, Colombia en el 2014, utilizando información registrada en resultados de laboratorio e historias clínicas, análisis efectuado mediante el programa EPI INFO versión 7. Resultados: Se analizaron 600 pacientes, el promedio de edad de la población fue de 59 años, el $65.4 \%$ fueron mujeres. La frecuencia de hipercolesterolemia fue $86.28 \%$, hipertrigliceridemia $68.35 \%$, colesterol HDL bajo $32.64 \%$ y dislipidemia mixta $33.33 \%$. Los principales factores de riesgo identificados fueron sobrepeso (44.67\%), hipertensión arterial (39.8\%), obesidad $(26.67 \%)$, y diabetes mellitus tipo 2 (13.17\%). Conclusiones: La dislipidemia es un factor de riesgo cardiovascular de gran impacto en salud pública lo cual incentiva a desarrollar y fortalecer estrategias de prevención y tratamiento oportuno.

Palabras Clave: Dislipidemia, hipercolesterolemia, hipertrigliceridemia.
\end{abstract}

\section{ABSTRACT}

Introduction: Dyslipidemia constitutes a major and modifiable risk factor in the genesis of cardiovascular diseases. Objective: Characterize adult patients with dyslipidemia who came to the outpatient service of the Valle de Tenza Regional Hospital, Guateque Boyaca, Colombia, in 2014. Material and method: A retrospective cross-sectional observational study was conducted. Patients aged 18 or over with alterations in the lipid profile were included in follow-up by outpatient consultation at the Valle de Tenza Regional Hospital, Guateque, Boyaca, Colombia in 2014, using information from laboratory results and medical records. The analysis was carried out using the software "EPI INFO", version 7. Results: 600 patients were analyzed, the average age of the population was 59 years, $65.4 \%$ were women. The frequency of hypercholesterolemia was $86.28 \%$, hypertriglyceridemia $68.35 \%$, elevated LDL cholesterol $73.72 \%$, low HDL cholesterol $32.64 \%$ and combined dyslipidemia $33.33 \%$. The main risk factors found were overweight (44.67\%), hypertension (39.8\%), obesity (26.67\%), and type 2 diabetes mellitus (13.17\%). Conclusions: Dyslipidemia is a cardiovascular risk factor with a great impact on public health, which encourages the development and strengthening of prevention and timely treatment strategies.

Key words: Dyslipidemia, hypercholesterolemia, hypertriglyceridemia. 


\section{Introducción}

La dislipidemia es un conjunto de alteraciones en las concentraciones de los lípidos o componentes de las lipoproteínas circulantes a nivel sanguíneo. La dislipidemia constituye un factor de riesgo mayor y modificable en la génesis de las enfermedades cardiovasculares ateroscleróticas (Jellinger, y otros, 2017). Los niveles séricos de colesterol LDL corresponden a la forma dominante de colesterol aterogénico y representan una causa primaria de ateroesclerosis, por lo tanto, las reducciones en sus concentraciones sanguíneas generan gran reducción en el riesgo de Enfermedad Cardiovascular Aterosclerótica (Grundy, y otros, 2019). Las concentraciones séricas de colesterol y triglicéridos y su metabolismo están determinados tanto por componentes genéticos de la persona como por factores adquiridos y modificables del estilo de vida, la dieta, y el nivel de actividad física (Teresich, 2014). La Organización Mundial de la Salud (OMS) recomienda un diagnóstico temprano y tratamiento oportuno para las personas con enfermedad cardiovascular o consideradas con alto riesgo cardiovascular, dado por la presencia de uno o más factores de riesgo como hipertensión arterial, dislipidemia, diabetes mellitus, o alguna enfermedad cardiovascular ya establecida (Organización Mundial de la Salud, 2017).

Las enfermedades cardiovasculares encabezan las causas de mortalidad a nivel mundial. Se estima que, en el año 2015, 17.7 millones de personas en el mundo murieron por esta causa, correspondiendo al $31 \%$ de los registros de mortalidad (Organización Mundial de la Salud, 2017). Según la OMS, la mayoría de los pacientes que padecen de hipercolesterolemia en el mundo no reciben tratamiento para contrarrestar esta enfermedad, generalmente porque desconocen que necesitan tratamiento, a pesar de que los medicamentos hipolipemiantes se encuentran fácilmente disponibles para su uso, además de su bajo costo y alta eficacia (Organización Mundial de la Salud, 2011).

Según la Organización Panamericana de la Salud, en Colombia en el 2008, las enfermedades isquémicas del corazón fueron la principal causa de mortalidad en hombres y mujeres mayores de 45 años, y las enfermedades cerebrovasculares constituyeron el tercer lugar del total de muertes en los hombres mayores de 45 años, y el segundo en las mujeres de esta misma edad (Organización Panamericana de la Salud, 2010).

A nivel global, la prevalencia de dislipidemia varía. En el estudio EURIKA, que incluyó población europea, se encontró prevalencia de hipertrigliceridemia en $20.8 \%$ y niveles bajos de colesterol HDL en $22.1 \%$ de los participantes (Halcox, et al., 2017). En Sudáfrica, el estudio HAALSI reportó que el $67.3 \%$ de los pacientes con reportes de perfil lipídico cumplieron criterios de dislipidemia, y se encontró que la hipertrigliceridemia fue el hallazgo más frecuente (Reiger, y otros, 2017). Por otra parte, en China del Norte registraron una prevalencia general de dislipidemia de $31.2 \%$, donde $4.3 \%$ tenía niveles séricos de colesterol total elevado, $2.4 \%$ colesterol LDL elevado, $14.7 \%$ hipertrigliceridemia y $17.4 \%$ colesterol HDL bajo (Xi, et al., 2020). Los registros en Norteamérica muestran que el $45 \%$ de los canadienses entre los 18 a 79 años padecen de dislipidemia (Joffres, Shields, Tremblay, \& ConnorGorber, 2013), mientras que, en Estados Unidos, durante el 2015 a 2016 , el $12.4 \%$ y el $18 \%$ de los adultos tenían colesterol total elevado y concentraciones séricas de colesterol HDL bajo, respectivamente (Carroll, Fryar, \& Nguyen, 2017). En cuanto a Latinoamérica, en el estudio CESCAS, una cohorte prospectiva realizada en 4 ciudades ( 2 en Argentina, una en Chile y una en Uruguay), se observó una prevalencia de dislipidemia en el 58.4\% de la población (Rubinstein, et al., 2015). La Encuesta Nacional de Salud, Chile 2016 - 2017 reporta HDL bajo en $45.8 \%$, LDL elevado en $5.2 \%$, hipertrigliceridemia en $35.8 \%$, colesterol total elevado en $27.8 \%$ de la población (Margozzini \& Passi, 2018).

En Colombia, en el 2009 se aprobó la Ley 1355 "por medio de la cual se define la obesidad y las enfermedades crónicas no transmisibles asociadas a esta como una prioridad de salud pública y se adoptan medidas para su control, atención y prevención", resaltando la importancia de la modificación de los estilos de vida hacia una vida saludable (Instituto Colombiano de Bienestar Familiar, 2009). En el Plan Decenal de Salud Pública 2012-2021, se encuentra como una de las metas la reducción relativa del $20 \%$ de los niveles de colesterol elevado en las personas de 18 años y más 
(Ministerio de Salud y Protección Social (Colombia), 2013), dado que es una de las prioridades para mejorar la situación de salud del país.

En nuestra población de estudio, a pesar de tratarse de un departamento en Colombia con alta carga de enfermedad cardiovascular, se desconocen datos epidemiológicos sobre dislipidemia. Este es el primer estudio en la región que describe la frecuencia de los tipos de dislipidemia y los factores de riesgo relacionados, siendo de gran importancia dado que incentiva a que se prioricen intervenciones enfocadas a la prevención primaria y secundaria, diagnóstico temprano y tratamiento oportuno de la dislipidemia en este grupo poblacional y que genere un impacto en salud pública. El objetivo de este trabajo es caracterizar los pacientes adultos con dislipidemia que consultan al servicio ambulatorio del Hospital Regional Valle de Tenza, Guateque Boyacá, Colombia, en el año 2014.

\section{Material y Método}

Diseño:

Se llevó a cabo un estudio observacional de corte transversal retrospectivo.

Población de estudio:

Se incluyeron todos los pacientes de 18 o más años que fueron atendidos en consulta ambulatoria del Hospital Regional Valle de Tenza del municipio de Guateque, Boyacá, Colombia, entre el periodo del 1 de enero y el 31 de diciembre del año 2014, que presentaron alguna alteración de los componentes del perfil lipídico identificado a partir de la revisión de resultados de laboratorio clínico.

El municipio de Guateque es la capital de la Provincia del Oriente, situado en el suroeste del departamento de Boyacá, a una distancia de $112 \mathrm{~km}$ de Bogotá, la capital del país. Cuenta con 9603 habitantes (DANE, 2015) (Alcaldía Municipal de Guateque, 2016). La economía de este municipio y de la región está basada principalmente en la actividad agrícola. Entre el año 2005 y 2014, las principales causas de mortalidad identificadas en el municipio de Guateque, tanto en hombres como en mujeres, fueron la enfermedad isquémica del corazón en el primer lugar, seguida por las enfermedades cerebrovasculares en el segundo lugar (Mendoza Torres, 2016).
Criterios de inclusión: adultos con dislipidemia que acudieron a consulta externa y disponían de expediente clínico y que incluyera en él, reportes de pruebas de laboratorio clínico de perfil lipídico, con seguimientos médicos que permitieran evaluar todas las variables del presente estudio. Se excluyeron los pacientes sin control médico durante el año 2014 y aquellos con historia clínica incompleta, que no proporcionaran los datos necesarios para estimar las variables requeridas para este estudio. Se documentó un total de 1305 pacientes con reportes de laboratorio clínico de perfil lipídico alterado; de estos, solo se encontraron expedientes con historias clínicas completas y con seguimiento de controles médicos de 600 expedientes.

Variables de estudio:

Se analizaron variables sociodemográficas (sexo, edad (años), procedencia), antecedentes personales de estos se consideraron: patológicos (diagnóstico de hipertensión arterial, diabetes mellitus, enfermedad renal crónica, obesidad, sobrepeso, síndrome de ovario poliquístico, hiperuricemia, hipotiroidismo, colestasis), tóxicos (consumo de alcohol, tabaquismo) y farmacológicos (uso de fármacos como tiazidas o estrógenos), valoración médica (índice de masa corporal (IMC) y diagnóstico actual), reportes de laboratorio clínico: perfil lipídico (resultados de niveles séricos de colesterol total, colesterol HDL, colesterol LDL, y triglicéridos en $\mathrm{mg} / \mathrm{dl}$ ) y tratamiento médico implementado para control de la enfermedad.

Los criterios que se consideraron en este estudio para categorizar el tipo de dislipidemia siguieron las recomendaciones de las guías ATP III, 2002. Según las guías ATP III (National Cholesterol Education Program (NCEP) Expert Panel on Detection, Evaluation, and Treatment of High Blood Cholesterol in Adults (Adult Treatment Panel III), 2002), las alteraciones en el perfil lipídico se clasificaron de la siguiente manera:

- Colesterol LDL elevado: una concentración de colesterol ligado a lipoproteínas de baja densidad (cLDL) igual o mayor a $100 \mathrm{mg} / \mathrm{dl}$, o según factores de riesgo individuales.

- Hipercolesterolemia: niveles séricos de colesterol total igual o mayor de $200 \mathrm{mg} / \mathrm{dl}$.

- Dislipidemia mixta: concentraciones de colesterol LDL y triglicéridos elevados. 
- Hipertrigliceridemia aislada: concentraciones de triglicéridos igual o mayor de $150 \mathrm{mg} / \mathrm{dl}$.

- Colesterol HDL bajo: concentraciones de colesterol ligado a lipoproteínas de alta densidad (cHDL) inferiores a $40 \mathrm{mg} / \mathrm{dl}$.

Recolección de información: El grupo de investigación estructuró y presentó el protocolo de investigación en el año 2015, luego de obtener los avales correspondientes, se llevó a cabo la recolección de la información requerida para evaluar las variables del estudio, para lo cual se efectuó una revisión detallada de los resultados de laboratorio clínico y de las historias clínicas en físico de los pacientes con alteraciones en el perfil lipídico en el año 2014 (1 de enero al 31 de diciembre) del Hospital Regional de Valle de Tenza de Guateque, Boyacá, Colombia.

Análisis estadístico:

Se llevó a cabo un análisis estadístico de tipo descriptivo presentando las variables cualitativas como frecuencias absolutas y relativas, y las variables cuantitativas a través de medidas de tendencia central (media) y de dispersión (desviación estándar) según comportamiento de distribución normal. El análisis fue realizado mediante el programa EPI INFO versión 7 (Centers for Disease Control and Prevention, 2019).

Consideraciones éticas:

El protocolo de estudio fue aprobado por el comité técnico directivo del Hospital Regional Valle de Tenza, para el análisis de la información de manera retrospectiva siguiendo los lineamientos nacionales e internacionales de bioética e investigación en seres humanos y garantizando el manejo confidencial de la información de los pacientes contenida en los expedientes clínicos.

\section{Resultados}

El promedio de edad de los sujetos de estudio fue de 59 años \pm 15.85 , en su mayoría de sexo femenino $(65.4 \%, n=392)$ y procedentes de Guateque $(53.67 \%, \mathrm{n}=322)$.

En cuanto a los tipos de alteraciones en el perfil lipídico identificados, la hipercolesterolemia fue el hallazgo más frecuente en esta población, seguido por el colesterol LDL elevado (Tabla 1).
Tabla 1. Frecuencia de alteraciones en el perfil lipídico

\begin{tabular}{lcc}
\hline \multicolumn{1}{c}{ Tipo de alteración } & $\mathrm{n}$ & $\%$ \\
\hline & & \\
Hipercolesterolemia & 518 & 86.28 \\
Colesterol LDL elevado & 442 & 73.72 \\
Hipertrigliceridemia & 410 & 68.35 \\
Dislipidemia mixta & 200 & 33.33 \\
Colesterol HDL bajo & 196 & 32.64 \\
\hline N: 600 & & \\
Fuente: Revisión de resultados de laboratorio clínico en físico
\end{tabular}

También se evaluaron variables consideradas en literatura como factores de riesgo para dislipidemia, encontrando en mayor frecuencia factores como sobrepeso, hipertensión arterial, obesidad y diabetes mellitus tipo 2 (Tabla 2).

Tabla 2. Frecuencia de factores de riesgo presentes para dislipidemia en la población de estudio

\begin{tabular}{lcc}
\hline \multicolumn{1}{c}{ Factor de riesgo } & $\mathrm{n}$ & $\%$ \\
\hline Consumo de Alcohol & 4 & \\
Uso de estrógenos & 6 & 0.67 \\
Uso de hidroclorotiazida & 72 & 1.00 \\
Sobrepeso & 268 & 44.67 \\
Obesidad & 160 & 26.67 \\
Hipertensión arterial & 239 & 39.80 \\
Diabetes Mellitus Tipo 2 & 79 & 13.17 \\
Enfermedad renal crónica & 18 & 3.00 \\
Hipotiroidismo & 44 & 7.33 \\
Hiperuricemia & 4 & 0.67 \\
Síndrome de ovario Poliquístico & 3 & 0.50 \\
Colestasis & 1 & 0.17 \\
\hline
\end{tabular}

$\mathrm{N}=600$

Fuente: Revisión de historias clínicas en físico

En cuanto al tratamiento médico para la dislipidemia en la población de estudio, el $27.67 \%(n=166)$ de la población recibió tratamiento no farmacológico; y del manejo farmacológico prescrito, el medicamento más utilizado fue la atorvastatina $(25.8 \% \mathrm{n}=155)$ (Figura 1). 


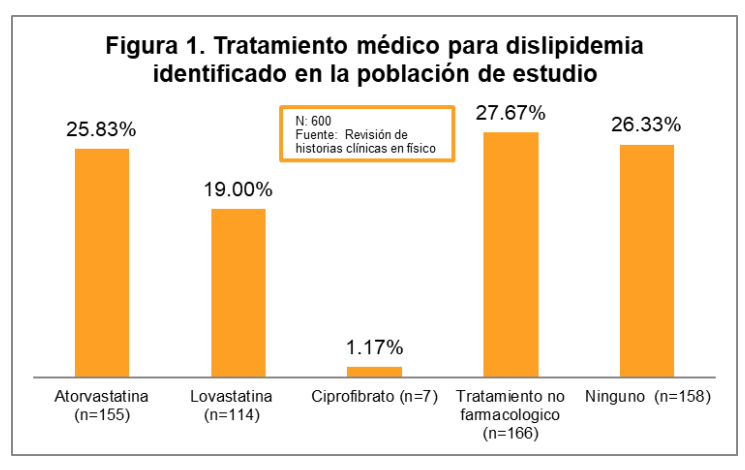

\section{Discusión}

Se estudiaron 600 pacientes con alteraciones en el perfil lipídico, encontrando que la mayoría fueron mujeres, siendo la hipercolesterolemia el tipo de alteración de perfil lipídico más prevalente, los factores de riesgo asociados con mayor frecuencia fueron el sobrepeso y la hipertensión arterial, y como manejo farmacológico principal se evidenció la prescripción de atorvastatina.

La dislipidemia es un problema de salud pública por su alta participación en el desarrollo y progreso de la enfermedad cardiovascular; el estudio CARMELA (Cardiovascular Risk Factor Multiple Evaluation in Latin America) mostró en sus resultados que la edad promedio de los pacientes con riesgo metabólico en Bogotá fue de 45.1 años (Schargrodsky, y otros, 2008), mientras que en nuestro estudio el promedio de edad en pacientes con dislipidemia fue de 59 años, cifra que se relaciona con el intervalo de edad de 45 a 65 años en los que las enfermedades isquémicas del corazón y las enfermedades cerebrovasculares representan las primeras causas de mortalidad en Colombia (Organización Panamericana de la Salud, 2010). Se encontró en este estudio que el $65.4 \%$ de los pacientes con dislipidemia fueron mujeres, así como lo observado en un hospital de la provincia de Azuay-Ecuador (Palacio Rojas, y otros, 2017) y en un estudio realizado en cuatro ciudades colombianas (Pereira, Manizales, Armenia y Cartago) (Machado Alba J. E., Machado Duque, Yepes, Manrique, \& Tobón, 2016) en los que reportan predominio de dislipidemia en mujeres, lo que puede estar relacionado con un mayor porcentaje de grasa corporal total en las mujeres en comparación con los hombres (Centers for Disease Control and Prevention, 2008) y mayor frecuencia de exceso de peso en las mujeres (Organización Mundial de la Salud, 2020; Bays, y otros, 2013).
Según Machado (Machado Alba \& Machado Duque, 2013), la dislipidemia mixta, hipercolesterolemia aislada e hipertrigliceridemia se reportaron en $46.6 \%, 29.4 \%$ y $20.3 \%$ respectivamente; Durango G (Durango Villadiego \& Corredor Pereira, 2013) reportó una prevalencia de $50 \%$ de hipercolesterolemia aislada y $53.3 \%$ de hipertrigliceridemia, mientras que los resultados en una IPS en Medellín, Colombia, han registrado una prevalencia de hipertrigliceridemia del $41.8 \%$, hipercolesterolemia 46\%, dislipidemia $74.7 \%$ (Galvis Pérez, Barona Acevedo, \& Cardona Arias, 2016). En cuanto a los hallazgos de alteraciones en el perfil lipídico en el presente estudio, dislipidemia mixta se presentó en el $33.33 \%$, hipercolesterolemia en el $86.28 \%$ e hipertrigliceridemia en el $68.35 \%$. Estos resultados muestran que la población estudiada presenta mayor frecuencia de hipercolesterolemia, alteración lipídica que se considera un factor de riesgo mayor para el desarrollo de enfermedades cardiovasculares ateroscleróticas (Jellinger, y otros, 2017), lo que pudiera estar contribuyendo a las principales causas de mortalidad identificadas en el municipio de Guateque, la enfermedad isquémica del corazón y las enfermedades cerebrovasculares (Mendoza Torres, 2016).

Se han reportado una serie de patologías crónicas no transmisibles como factores de riesgo para el desarrollo de la dislipidemia. El estudio HISPALIPID (Vegazo, y otros, 2006) reportó que el $86.4 \%$ de los pacientes con dislipidemia presentaron algún otro factor de riesgo cardiovascular, la comorbilidad más frecuentemente asociada a este problema fue la hipertensión arterial (51.1\%). Machado encontró diabetes mellitus en $28.5 \%$ e hipertensión arterial en $93.2 \%$ de los pacientes con dislipidemia (Machado Alba \& Machado Duque, 2013). En un estudio realizado en estudiantes universitarios en Bogotá, Colombia encontraron una asociación significativa entre la obesidad y la hipertrigliceridemia (Almonacid Urrego, y otros, 2016). En el estudio realizado por Zuluaga-Quintero et al, en los pacientes con dislipidemia tratados con estatinas en una IPS se presentaron comorbilidades similares a las encontradas en el presente estudio tales como hipertensión arterial, diabetes mellitus y enfermedad tiroidea (Zuluaga Quintero, Arcila Hincapie, Bedoya López, Toro Palacio, \& Arboleda Velásquez, 2015). En el presente estudio el $13.17 \%$ 
presentaban la comorbilidad de diabetes mellitus, el $39.8 \%$ hipertensión arterial, y el $26.67 \%$ eran obesos. Lo anterior resalta como la dislipidemia está altamente relacionada con otras condiciones de riesgo cardiovascular modificables y de gran impacto en salud pública, que al ser tratada de manera oportuna y adecuada pudieran ayudar a una disminución del riesgo cardiovascular.

Existe una relación fisiopatológica para el desarrollo de dislipidemia en pacientes que padecen de obesidad, debido a que los adipocitos cumplen funciones endocrinas e inmunes, y al presentarse una acumulación excesiva de tejido adiposo se genera una elevación anormal de los niveles de lípidos circulantes (Bays, y otros, 2013). En el 2010, en la Encuesta Nacional de la Situación Nutricional en Colombia se reportó un aumento progresivo del exceso de peso, pasando del $45.9 \%$ de la población en el año 2005 a $51.2 \%$ en el 2010; así mismo se reportó que el exceso de peso era más frecuente en las mujeres que en los hombres $(55.2 \%$ frente a 45.6\%) (Instituto Colombiano de Bienestar Familiar, 2010), similar a lo observado en otros países de América Latina (Organización Panamericana de Salud, 2017), y a lo valorado en 2016 a nivel mundial en la población adulta, donde un $39 \%$ de los hombres y un $40 \%$ de las mujeres tenían sobrepeso, y un $11 \%$ de los hombres y un $15 \%$ de las mujeres padecían algún grado de obesidad (Organización Mundial de la Salud, 2020). En el presente estudio, el $71 \%$ de los pacientes tuvieron exceso de peso (sobrepeso y obesidad), cifra que demuestra una alta frecuencia de presentación concomitante de estas patologías en esta población, lo que indicaría que los estilos de vida de esta población son poco saludables y explicaría la alta prevalencia de hipercolesterolemia e hipertrigliceridemia reportada.

En el presente estudio el $46 \%$ tenían tratamiento farmacológico, los medicamentos más utilizados fueron las estatinas y en menor proporción los fibratos, $27.67 \%$ de los pacientes fueron tratados solamente con medidas no farmacológicas, pero no se pudo determinar qué tipo de recomendaciones se le indicaba al paciente y en un $26 \%$ no se determinó ningún tratamiento. El estudio HISPALIPID reporta un $72.6 \%$ de pacientes tratados con algún medicamento hipolipemiante, siendo las estatinas el medicamento que recibió la mayoría de los participantes del estudio (Vegazo, y otros, 2006). Si bien el porcentaje de pacientes que recibieron algún manejo farmacológico fue mayor en el estudio HISPALIPID en comparación con el nuestro, en ambos estudios el manejo farmacológico más frecuente se encuentra acorde con el tratamiento de primera línea para la hipercolesterolemia. La estatina más prescrita en nuestro estudio fue la atorvastatina, siendo esta la intervención más costo-efectiva para pacientes con dislipidemia con predominio de hipercolesterolemia y que tengan criterios que indiquen tratamiento farmacológico con terapia moderada y alta con estatinas en Colombia, dado la ganancia en años de vida ajustados por calidad (AVAC) y su bajo costo (Rosselli, 2015).

Es importante conocer las diferentes opciones y respuesta terapéutica del manejo farmacológico de la dislipidemia, entre los que se encuentran las estatinas, con las que se logra una disminución de la concentración sérica de colesterol LDL en 18-55\%, aumento del colesterol HDL en 5-15\% y disminución del 7-30\% en los niveles séricos de triglicéridos. Los fibratos generan una disminución de los niveles séricos de triglicéridos entre 20 a $50 \%$ y del colesterol LDL en 5 a $20 \%$ y un aumento del colesterol HDL en 10 a $35 \%$. Otro hipolipemiante, la niacina, aumenta las concentraciones de colesterol HDL en 15 a $25 \%$, disminuye los niveles séricos de colesterol LDL en 5 a $25 \%$, y reduce los triglicéridos séricos en 20 a $50 \%$. Ezetimiba disminuye el colesterol LDL en $18-20 \%$ y triglicéridos en $5-14 \%$ y aumenta el colesterol HDL en 1 a 5\% (Juan López, y otros, 2013). Lo anterior resalta que las estatinas son los medicamentos de elección para lograr mayor disminución de los niveles séricos de colesterol LDL y así reducir el riesgo de enfermedades cardiovasculares ateroscleróticas (Grundy, y otros, 2019).

Ya implementado el tratamiento y teniendo en cuenta las guías del ATP III, las recomendaciones deben dirigirse a realizar un control de perfil lipídico en seis semanas posterior al inicio de la terapia farmacológica y continuar cada seis semanas hasta alcanzar el objetivo (National Cholesterol Education Program (NCEP) Expert Panel on Detection, Evaluation, and Treatment of High Blood Cholesterol in Adults (Adult Treatment Panel III), 2002). Posteriormente realizar un control cada seis a doce meses. 
Es de vital importancia implementar medidas tan trascendentales como las no farmacológicas, incentivando a la población del Valle de Tenza a disminuir de peso, adoptando estilos de vida saludables con modificaciones en la dieta, realizar ejercicio físico regular, disminuir el consumo de alcohol y el tabaquismo, y logrando un adecuado control de las comorbilidades, las cuales además aumentan el riesgo cardiovascular. Dentro de las recomendaciones dietarias se encuentran reducir el consumo de grasas saturadas, ya que estas aumentan los niveles séricos de colesterol LDL, prefiriendo las grasa insaturadas y entre estas las monoinsaturadas que disminuyen las concentraciones séricas de colesterol LDL y al mismo tiempo generan un factor protector aumentado el colesterol HDL, adicionalmente una dieta baja en calorías, rica en verduras y frutas, reducir la ingesta de carbohidratos y aumentar el consumo de fibra soluble (Juan López, y otros, 2013). Motivar la práctica de actividad física aeróbica regular (Arizmendi, 2010); en la población adulta con dislipidemia se sugiere realizar ejercicio físico de resistencia cardiovascular entre tres a cinco veces a la semana, cada sesión con una duración de 20 a 60 minutos, con intensidad moderada a vigorosa, actividad física que debe ser indicada de manera individual dependiendo las características particulares de cada paciente (Ministerio de Salud y Protección Social - Colciencias, 2014). Es importante destacar que el tratamiento no farmacológico como disminución de peso, actividad física y abandono del cigarrillo pueden aumentar el colesterol HDL de 5 a 20\%, 5 a $30 \%$ y 5\%, respectivamente (Juan López, y otros, 2013).

\section{Limitaciones del estudio}

Se encontró alteraciones en algún componente del perfil lipídico en los resultados del laboratorio del Hospital Regional Valle de Tenza del municipio de Guateque, Boyacá, Colombia, entre el 1 de enero y el 31 de diciembre de 2014 en 1305 pacientes, sin embargo, se presentó la limitación que solo se encontraron historias clínicas completas y con seguimiento de controles médicos a 600 de dichos pacientes, lo que se explica dado que esta institución es el hospital de segundo nivel de referencia para la comunidad de varios municipios que asisten a la realización de sus paraclínicos y continúan sus controles médicos en sus centros de atención primaria y otros por inasistencias a citas médicas de seguimiento. También como limitante se puede señalar que al ser un estudio descriptivo no se pueden realizar asociaciones estadísticas en causalidad entre los tipos de dislipidemia descritos y los factores de riesgos asociados en nuestra población.

\section{Conclusiones}

El presente estudio analizó una población de 600 pacientes adultos con dislipidemia atendidos de forma ambulatoria en un hospital regional de Colombia; la hipercolesterolemia y los niveles séricos de colesterol LDL fueron los tipos predominantes de dislipidemia encontrados, ambos conocidos por su relación con las enfermedades cardiovasculares ateroscleróticas. Las condiciones de riesgo más frecuentes fueron sobrepeso, hipertensión arterial, obesidad y diabetes mellitus tipo 2, también se encontró en algunos casos ausencia de tratamiento farmacológico. Dada la alta prevalencia de enfermedades cardiovasculares en la región, es indispensable implementar programas de búsqueda activa y seguimiento a los pacientes con dislipidemia acompañado de asesorías nutricionales y de educación terapéutica, estimulando la adherencia al tratamiento farmacológico y no farmacológico de manera que se generen procesos preventivos con enfoque en factores modificables que tengan un impacto en salud pública.

\section{Agradecimientos}

Los autores agradecen a las directivas del Hospital Regional Valle de Tenza de Guateque, Boyacá del año 2015 y al programa de Epidemiologia de la Fundación Universitaria Juan N. Corpas.

\section{Bibliografía}

Alcaldía Municipal de Guateque. (2016). Plan de Desarrollo 2016-2019. Boyacá, Guateque.

Almonacid Urrego, C. C., Camarillo Romero, M., Gil Murcia, Z., Medina Medina, C. Y., Rebellón Marulanda, J. V., \& Mendieta Zerón, H. (2016). Evaluación de factores de riesgo asociados a enfermedad cardiovascular en jóvenes universitarios de la Localidad Santafé en Bogotá, Colombia. NOVA, 14(25), 9-17. http://www.scielo.org.co/scielo.php?script=sci_a rttext\&pid=S1794$24702016000100004 \& \operatorname{lng}=$ en\&tlng=es

Arizmendi, G. M. (2010). Atención farmacéutica y nutricional a pacientes con dislipidemia. Las 
Tesinas de Belgrano, 443. Obtenido de https://biblioteca.ub.edu.ar/cgi-bin/koha/opacdetail.pl?biblionumber $=46757$

Bays, H. E., Toth, P. P., Kris Etherton, P. M., Abate, N., Aronne, L. J., Brown, W. V., . . Samuel, V. T. (2013). Obesity, adiposity, and dyslipidemia: a consensus statement from the National Lipid Association. Journal of clinical lipidology, 7(4), 304-383.

https://doi.org/10.1016/j.jacl.2013.04.001

Carroll, M., Fryar, C., \& Nguyen, D. (2017). Total and High-density Lipoprotein Cholesterol in Adults: United States, 2015-2016. NCHS data brief(290), 1-8.

Centers for Disease Control and Prevention. (30 de 12 de 2008). QuickStats: Mean Percentage Body Fat, * by Age Group and Sex --- National Health and Nutrition Examination Survey, United States, 1999--2004. Obtenido de https://www.cdc.gov/mmwr/preview/mmwrhtml /mm5751a4.htm

Centers for Disease Control and Prevention. (03 de 01 de 2019). Epi Info ${ }^{\text {TM }}$ For Windows. Obtenido de

https://www.cdc.gov/epiinfo/support/downloads. html

Durango Villadiego, G., \& Corredor Pereira, C. (2013). Prevalencia de dislipidemias en adultos atentidos en la unidad especial de salud de la universidad de Córdoba (Tesis de Maestría ed.). Cartagena de Indias: Universidad San Buenaventura. Obtenido de http://bibliotecadigital.usb.edu.co/bitstream/108 19/2373/1/Prevalencia\%20de\%20dislipidemias \%20en\%20adultos_Germ\%C3\%A1n\%20Duran go_USBCTG_2014.pdf

Galvis Pérez, Y., Barona Acevedo, J., \& Cardona Arias, J. (2016). Prevalencia de dislipidemias en una institución prestadora de servicios de salud de Medellín (Colombia), 2013. CES Medicina, $30(1)$, 3-13. https://www.redalyc.org/articulo.oa?id=2611/26 1146219002
Grundy, S., Stone, N., Bailey, A., Beam, C., Birtcher, K., Blumenthal, R., . . Y Yeboah, J. (2019). 2018 AHA/ACC/AACVPR/AAPA/ABC/ACPM/AD A/AGS/APhA/ASPC/NLA/PCNA Guideline on the Management of Blood Cholesterol: A Report of the American College of Cardiology/American Heart Association Task Force on Clinical Practice Guidelines. Journal of the American College of Cardiology, 73(24), e285-e350. https://doi.org/10.1016/j.jacc.2018.11.003

Halcox, J., Banegas, J., Roy, C., Dallongeville, J., De Backer, G., Guallar, E., . . . Borghi, C. (2017). Prevalence and treatment of atherogenic dyslipidemia in the primary prevention of cardiovascular disease in Europe: EURIKA, a cross-sectional observational study. $B M C$ cardiovascular disorders, 17(1), 160. https://doi.org/10.1186/s12872-017-0591-5

Instituto Colombiano de Bienestar Familiar. (2009). Recuperado el 02 de 01 de 2020, de Ley 1355 de 2009:

https://www.icbf.gov.co/cargues/avance/docs/le y_1355_2009.htm

Instituto Colombiano de Bienestar Familiar. (2010). Recuperado el 02 de 02 de 2020, de Encuesta Nacional de la Situación Nutricional en Colombia 2010, ENSIN: https://www.icbf.gov.co/sites/default/files/resum enfi.pdf

Jellinger, P. S., Handelsman, Y., Rosenblit, P. D., Bloomgarden, Z. T., Fonseca, V. A., Garber, A. J., . . . Davidson, M. (2017). AMERICAN ASSOCIATION OF CLINICAL ENDOCRINOLOGISTS AND AMERICAN COLLEGE OF ENDOCRINOLOGY GUIDELINES FOR MANAGEMENT OF DYSLIPIDEMIA AND PREVENTION OF CARDIOVASCULAR DISEASE. Endocrine practice : official journal of the American College of Endocrinology and the American Association of Clinical Endocrinologists, 23(Suppl 2), 1-87. https://doi.org/10.4158/EP171764.APPGL

Joffres, M., Shields, M., Tremblay, M., \& ConnorGorber, S. (2013). Dyslipidemia prevalence, treatment, control, and awareness in the Canadian 
Health Measures Survey. Canadian journal of public health $=$ Revue canadienne de sante publique, 104(3), e252-e257. https://doi.org/10.17269/cjph.104.3783

Juan López, M., Kuri Morales, P., Durán Fontes, L., Velasco Gonzalez, M., Ruiz Palacios, G., Arriola Peñalosa, M. A., . . . Gonzalez Roldán, J. (Julio de 2013). Guía de Tratamiento Farmacológico de Dislipidemias para el primer nivel de atención. Revista mexicana de cardiología, 24(3), 103-129. http://www.scielo.org.mx/scielo.php?script=sci_ arttext\&pid=S0188$21982013000300001 \& \operatorname{lng}=\mathrm{es} \& \operatorname{tlng}=\mathrm{es}$

Machado Alba, J. E., \& Machado Duque, M. E. (2013). Prevalencia de factores de riesgo cardiovascular en pacientes con dislipidemia afiliados al sistema de salud en Colombia. Revista Peruana de Medicina Experimental y Salud Publica, 30(2), 205-211. http://www.scielo.org.pe/scielo.php?script=sci_a rttext\&pid=S1726-

$46342013000200007 \& \operatorname{lng}=\mathrm{es} \& \ln \mathrm{ln}=\mathrm{es}$

Machado Alba, J. E., Machado Duque, E., Yepes, M. C., Manrique, S., \& Tobón, L. M. (2016). Tratamiento hipolipemiante y su efectividad en pacientes de cuatro ciudades colombianas. Acta Medica Colombiana, 41(3), 181-186. http://www.scielo.org.co/scielo.php?script=sci_a rttext\&pid=S0120-

24482016000300181\&lng=en\&tlng=es

Margozzini, P., \& Passi, A. (2018). Encuesta Nacional de Salud, ENS 2016-2017: un aporte a la planificación sanitaria y políticas públicas en Chile. ARS MEDICA Revista De Ciencias Médicas, 43(1), 30-34. https://doi.org/10.11565/arsmed.v43i1.1354

Mendoza Torres, L. E. (2016). Análisis de Situación de Salud con el Modelo de los Determinantes Sociales de Salud. ESE Hospital Regional Valle de Tenza, Boyacá, Guateque.

Ministerio de Salud y Protección Social Colciencias. (2014). Guía de práctica clínica para la prevención, detección temprana, diagnóstico, tratamiento y seguimiento de las dislipidemias en la población mayor de 18 años. Bogotá.

Ministerio de Salud y Protección Social (Colombia). (2013). Plan Decenal de Salud Pública, PDSP, 2012 -2021. Recuperado el 01 de 03 de 2020, de Plan Decenal de Salud Pública2012 -2021: https://www.minsalud.gov.co/Documentos\%20y \%20Publicaciones/Plan\%20Decenal\%20-

$\% 20$ Documento $\% 20$ en $\% 20$ consulta $\% 20$ para $\% 2$ 0aprobaci\%C3\%B3n.pdf

National Cholesterol Education Program (NCEP) Expert Panel on Detection, Evaluation, and Treatment of High Blood Cholesterol in Adults (Adult Treatment Panel III). (2002). Third Report of the National Cholesterol Education Program (NCEP) Expert Panel on Detection, Evaluation, and Treatment of High Blood Cholesterol in Adults (Adult Treatment Panel III) final report. Circulation, 106(25), 3143-3421. https://www.nhlbi.nih.gov/files/docs/resources/h eart/atp-3-cholesterol-full-report.pdf

Organización Mundial de la Salud. (2011). Recuperado el 01 de 03 de 2020, de El colesterol alto, un problema mal controlado: https://www.who.int/mediacentre/news/notes/20 11/cholesterol_20110201/es/

Organización Mundial de la Salud. (2017). Recuperado el 01 de 02 de 2020, de Enfermedades cardiovasculares: https://www.who.int/es/news-room/factsheets/detail/cardiovascular-diseases-(cvds)

Organización Mundial de la Salud. (2020). Recuperado el 10 de 04 de 2020, de Obesidad y sobrepeso: https://www.who.int/es/newsroom/fact-sheets/detail/obesity-and-overweight

Organización Panamericana de Salud. (2017). Recuperado el 15 de 01 de 2020, de Sobrepeso afecta a casi la mitad de la población de todos los países de América Latina y el Caribe salvo por Haití:

https://www.paho.org/col/index.php?option=co m_content\&view=article \&id=2686: sobrepesoafecta-a-casi-la-mitad-de-la-poblacion-de-todoslos-paises-de-america-latina-y-el-caribe-salvopor-haiti\&Itemid=562 
Organización Panamericana de la Salud. (2010). Indicadores Básicos 2010 : Situación de Salud en Colombia. Obtenido de https://www.paho.org/col/index.php?option=co m_docman\&view=download\&category_slug=da tos-y-estadisticas \&alias $=1195$-situacion-desalud-en-colombia-indicadores-basicos2010\&Itemid $=688$

Palacio Rojas, M., Nuñez Nava, T., García Pacheco, M., Capelo Ramón, C., Barahona Astudillo, M., Llumiquinga Yar, V., . . Rojas Páez, R. (2017). Prevalencia de dislipidemia y factores asociados en individuos adultos. Hospital básico de Paute, provincia de Azuay-Ecuador. Síndrome Cardiometabólico y enfermedades Crónico degenerativas, 7(1), 62-66.

Reiger, S., Veiga, T., Abrahams-Gessel, S., Crowther, N., Wade, A., Gomez-Olive, X., . . . Gaziano, T. (2017). Awareness, treatment, and control of dyslipidemia in rural South Africa: The HAALSI (Health and Aging in Africa: A Longitudinal Study of an INDEPTH Community in South Africa) study. PLoS ONE, 12(10). https://doi.org/10.1371/journal.pone.0187347

Rosselli, D. (2015). Costo-efectividad de las estatinas para el tratamiento de dislipidemia en Colombia. Acta Médica Colombiana, 40(2), 118124. https://doi.org/10.36104/amc.2015.461

Rubinstein, A., Irazola, V., Calandrelli, M., Elorriaga, N., Gutierrez, L., Lanas, F., . . He, J. (2015). Multiple cardiometabolic risk factors in the Southern Cone of Latin America: a population-based study in Argentina, Chile, and Uruguay. International journal of cardiology, 183,82-88.

https://doi.org/10.1016/j.ijcard.2015.01.062

Schargrodsky, H., Hernández-Hernández, R., Champagne, B. M., Silva, H., Vinueza, R., Silva Ayçaguer, L. C., . . . CARMELA Study Investigators. (2008). CARMELA: assessment of cardiovascular risk in seven Latin American cities. The American journal of medicine, 121(1), 58-65.

https://doi.org/10.1016/j.amjmed.2007.08.038
Teresich, R. L. (2014). Evaluación de la Alimentación en Pacientes Adultos con Dislipidemias (Tesis de Pregrado ed.). Rosario: Universidad Abierta Interamericana.

Vegazo, O., Banegas, J., Civeira, F., Serrano Aisa, P. L., Jiménez, F. J., \& Luengo, E. (Sep de 2006). Prevalencia de dislipemia en las consultas ambulatorias del Sistema Nacional de Salud: Estudio HISPALIPID. Medicina Clinica, 127(9).

Xi, Y., Niu, L., Cao, N., Bao, H., Xu, X., Zhu, H., . . . Zhang, X. (2020). Prevalence of dyslipidemia and associated risk factors among adults aged $\geq 35$ years in northern China: a cross-sectional study. BMC Public Health, 20(1068). https://doi.org/10.1186/s12889-020-09172-9

Zuluaga Quintero, N., Arcila Hincapie, L. M., Bedoya López, D. F., Toro Palacio, L. F., \& Arboleda Velásquez, C. (2015). Comportamiento del perfil lipídico en pacientes con dislipidemia tratados con estatinas en una IPS. CES Salud Pública, 6, 63-69. 